\title{
Value of diffusion-weighted magnetic resonance imaging in predicting World Health Organization grade in G1/G2 pancreatic neuroendocrine tumors
}

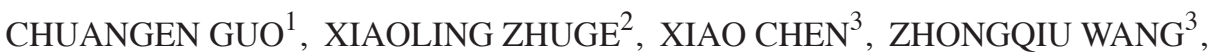 \\ WENBO XIAO $^{1}$ and QIDONG WANG ${ }^{1}$
}

\begin{abstract}
Departments of ${ }^{1}$ Radiology and ${ }^{2}$ Laboratory of Medicine, The First Affiliated Hospital, College of Medicine, Zhejiang University, Hangzhou, Zhejiang 310003; ${ }^{3}$ Department of Radiology, The Affiliated Hospital of Nanjing University of Chinese Medicine, Nanjing, Jiangsu 210029, P.R. China
\end{abstract}

Received March 17, 2016; Accepted February 17, 2017

DOI: $10.3892 / \mathrm{ol} .2017 .6029$

\begin{abstract}
The present study aimed to investigate the value of diffusion-weighted magnetic resonance imaging (DWI) in the grading of well-differentiated pancreatic neuroendocrine tumors (PanNETs). A total of 44 patients with histologically proven well-differentiated PanNET [grade 1 (G1) and grade 2 (G2) according to the World Health Organization (WHO) criteria] underwent pretreatment magnetic resonance imaging (MRI), which was retrospectively analyzed. The location, size, cystic or solid appearance, boundary, presence or absence of tumor contrast enhancement, and MRI signal of the tumor were assessed. Apparent diffusion coefficients (ADCs) within the primary tumor were measured on ADC maps. Receiver operating characteristic curves were used to determine ADC cut-off values and the sensitivity and specificity of the grade prediction. Spearman's rank correlation was utilized to probe the association between ADC value and PanNET grade or pathological parameters. G1 tumors exhibited a well-circumscribed border more often than G2 tumors. No marked differences were observed between PanNET G1 and PanNET G2 for cystic or solid appearance, enhancement, and T1- and T2-weighted imaging signals. Marked hyperintensities were more common in PanNET G2 tumors than in PanNET G1 tumors $(\mathrm{P}<0.01)$. The mean ADC values were statistically
\end{abstract}

Correspondence to: $\mathrm{Dr}$ Xiaoling Zhuge, Department of Laboratory of Medicine, The First Affiliated Hospital, College of Medicine, Zhejiang University, 79 Qingchun Road, Hangzhou, Zhejiang 310003, P.R. China

E-mail:xlzhuge@tom.com

Dr Chuangen Guo, Department of Radiology, The First Affiliated Hospital, College of Medicine, Zhejiang University, 79 Qingchun Road, Hangzhou, Zhejiang 310003, P.R. China

E-mail: zyyfy001@sina.cn

Key words: pancreatic neuroendocrine tumor, diffusion-weighted imaging, apparent diffusion coefficients, grade different between the normal pancreas and G1 and G2 tumors $(\mathrm{P}<0.01)$, and between PanNET G1 and PanNET G2 $(\mathrm{P}<0.05)$. Correlation analysis showed that ADC value was negatively correlated with PanNET grade, mitotic count and Ki-67 proliferation index $(\mathrm{P}<0.05)$. The cut-off $\mathrm{ADC}$ value was $0.930 \times 10^{-3} \mathrm{~mm}^{2} / \mathrm{sec}$, which identified G2 tumors with $82.4 \%$ sensitivity and $79.5 \%$ selectivity. ADC value therefore represents a non-invasive and valuable imaging parameter in predicting the WHO grade of tumors in well-differentiated PanNET.

\section{Introduction}

Pancreatic neuroendocrine tumors (PanNETs) are considered to be rare neoplasms, accounting for $1-2 \%$ of all pancreatic neoplasms (1). However, the diagnosed incidence of PanNET is increasing owing to the development of imaging technologies (2). PanNETs constitute a heterogeneous group of neoplasms (3) whose clinical and biological behavior ranges from benign to highly aggressive $(4,5)$.

The World Health Organization (WHO) has published several studies concerning the classification of neuroendocrine tumors (NETs) (6-8). A recent study classified NETs into NET grade $1(\mathrm{G} 1)$, NET grade $2(\mathrm{G} 2)$ or neuroendocrine carcinoma (NEC) based on pathological parameters, including the cell mitotic count and the Ki-67 proliferation index (8). NET G1 and G2 are well-differentiated tumor types, whereas NECs are poorly differentiated. G1 and G2 tumors exhibit different biological behaviors (1). Given that $\mathrm{G} 2$ tumors may possess more malignant features than G1 tumors, the pretreatment identification of NET grade is important in determining treatment strategy (9).

Numerous imaging methods have been employed for PanNET detection, including endoscopic ultrasound, computed tomography (CT), magnetic resonance imaging (MRI) and positron emission tomography (PET)/CT. The findings of PanNET imaging have been widely investigated; however, the biological features of a PanNET cannot be fully evaluated until the entire tumor has been resected (10). Recently, several studies have also tried to evaluate the grade and aggressiveness of PanNET using imaging techniques, including 
contrast-enhanced CT $(11,12)$, contrast-enhanced MRI (5), diffusion-weighted magnetic resonance imaging (DWI) (5) and fludeoxyglucose (FDG)-PET (13). These studies showed that these imaging techniques may be useful for differentiating benign PanNETs (G1 and G2) from non-benign PanNET (NEC). However, these studies did not show whether the imaging approach alone could differentiate between G1 and G2 tumors.

DWI is a functional modality that detects the random diffusion of water protons within biological tissues (14). It has been suggested that increased tumor cellularity causes a decrease in extracellular space, which may result in greater restriction of water diffusion (15). Recently, DWI has been used to predict the tumor staging, the tumor grade and the prognosis in a variety of neoplasms $(16,17)$. Tumor cellularity differs between PanNET G1 and PanNET G2. Thus, DWI could also be used to predict the PanNET grade. Based on 30 PanNET patients undergoing DWI scanning, Kim et al showed that DWI is useful for differentiating between PanNET G1 and PanNET G2 (2). However, to the best of our knowledge, no further studies have been performed on a larger population and the correlation between histopathological characteristics and apparent diffusion coefficient (ADC) has not been described. The aim of the present study was therefore to investigate the value of DWI in well-differentiated PanNET grading, and to identify the association between histopathological characteristics and ADC values.

\section{Materials and methods}

Patient selection. This retrospective study complied with the recommendations of our Institutional Review Board (The First Affiliated Hospital, College of Medicine Zhejiang University) and formal consent was waived. Between February 2012 and October 2014, 52 patients with proven PanNET (not including NEC) were considered for inclusion in this study. A total of 6 patients were excluded from this study owing to missing clinic records or DWI imaging sequences. In addition, 2 patients were excluded, as their tumors were not classified according to the WHO classification. A total of 44 patients with surgery-proven PanNET were finally evaluated in the study. In this study, PanNET was divided into NET G1 and NET G2 according to the updated WHO classification for neuroendocrine tumors (7).

MRI study. Upper abdominal MRI was performed using a Signa HDx 3.0-T unit (GE Healthcare Bio-Sciences, Pittsburgh, PA, USA) (n=26) or Achieva 3.0-T (Philips Medical Systems B.V., Eindhoven, The Netherlands) $(n=18)$ using eight-channel phased-array torso coils. Patients fasted for $8 \mathrm{~h}$ prior to MRI examination. Fat-suppressed liver acquisition with volume acceleration (GE Signa HDxt 3.0-T MRI unit; TR/TE, 3.1/1.5 msec) or 3D T1-weighted high-resolution isotropic volume examinations (Philips Achieva 3.0-T MRI unit; TR/TE, 4.0/1.9 msec) imaging sequences (with and without gadolinium) and a single-shot fast-spin-echo T2-weighted sequence (TR/TE, $5,000-8,000 / 80 \mathrm{msec}$ ), with a 4-5-mm slice thickness, $1-\mathrm{mm}$ interslice gap, $384 \times 256$ matrix and 30-35-cm field of view, were performed on each patient. DWI was performed using the single-shot echo-planar imaging technique (TR/TE,
Table I. Clinical data of patients.

\begin{tabular}{|c|c|}
\hline Variables & Value \\
\hline Mean age (range), years & $52.8(30-76)$ \\
\hline \multicolumn{2}{|l|}{ Gender, n (\%) } \\
\hline Male & $17(38.6)$ \\
\hline Female & $27(61.4)$ \\
\hline \multicolumn{2}{|l|}{ Clinical symptoms, n (\%) } \\
\hline Abdominal pain & $8(18.2)$ \\
\hline Confusion of consciousness & $5(11.4)$ \\
\hline Dizziness & $6(13.6)$ \\
\hline Hypodynamia & $3(6.8)$ \\
\hline Others & $5(11.4)$ \\
\hline Asymptomatic & $17(38.6)$ \\
\hline \multicolumn{2}{|l|}{ WHO classification, n (\%) } \\
\hline G1 & $34(77.3)$ \\
\hline $\mathrm{G} 2$ & $10(22.7)$ \\
\hline \multicolumn{2}{|l|}{ Location, n (\%) } \\
\hline Pancreatic head/neck & $23(52.3)$ \\
\hline Pancreatic body/tail & $21(47.7)$ \\
\hline Size (range), $\mathrm{cm}^{\mathrm{a}}$ & $2.4 \pm 1.6(0.8-6.5)$ \\
\hline $\mathrm{CEA}, \mathrm{ng} / \mathrm{ml}^{\mathrm{a}}$ & $5.4 \pm 19.9$ \\
\hline $\mathrm{CA} 199, \mathrm{ku} / \mathrm{l}^{\mathrm{a}}$ & $51.2 \pm 188.9$ \\
\hline Blood glucose, $\mathrm{mmol} / \mathrm{l}^{\mathrm{a}}$ & $4.9 \pm 1.37$ \\
\hline
\end{tabular}

${ }^{\text {aData }}$ are presented as the mean \pm standard deviation. WHO, World Health Organization; G, grade; CEA, carcinoembryonic antigen; CA19-9, cancer antigen 19-9.

$6,000-8,000 / 6-70 \mathrm{msec}$ ) with b-values of 0 and $1,000 \mathrm{sec} / \mathrm{mm}^{2}$. Following the intravenous injection of $0.1 \mathrm{mmol} / \mathrm{kg}$ gadolinium $(2.5 \mathrm{ml} / \mathrm{sec})$, axial and sagittal turbo-FLASH T1 images were obtained.

MRI analysis. Two experienced gastrointestinal radiologists (with >8 years of experience in abdominal MRI reading) were blinded to the clinical and pathological findings when analyzing the images. All images were reviewed on a picture archiving communication system and extended to an MR workstation for quantitative analysis. The imaging parameters included the tumor position, tumor size, tumor margin (well-circumscribed or ill-defined border), the presence of cystic components (solid or cystic-solid), and the presence or absence of enhancement. The presence of dilated pancreatic ducts and metastases was also recorded. Cystic lesions within the tumor were identified as areas that were hypointense on pre-contrast T1-weighted images, markedly hyperintense on T2-weighted images and presented with non-enhancement.

ADC maps of lesions were reconstructed at the workstation based on the b-values ( 0 and $1,000 \mathrm{sec} / \mathrm{mm}^{2}$ ). ADC values for the tumor and the normal pancreas were measured. A region of interest (ROI) was manually drawn along the tumor border in an image that showed the tumor at its maximum dimension, avoiding necrotic or cystic components. A similar ROI was manually drawn in the normal pancreas, avoiding the main 
Table II. Summary of MRI findings.

Tumor grade, $\mathrm{n}$

\begin{tabular}{|c|c|c|c|c|}
\hline \multirow{2}{*}{ MRI findings } & & & & \multirow{2}{*}{ P-value } \\
\hline & PanNET G1 $(n=34)$ & PanNET G2 $(n=10)$ & Total & \\
\hline Shape & & & & 0.01 \\
\hline Well-circumscribed & 34 & 7 & 40 & \\
\hline Ill-defined borders & 0 & 3 & 4 & \\
\hline Solid and cystic pattern & & & & 0.51 \\
\hline Solid & 5 & 2 & 7 & \\
\hline Cystic-solid & 29 & 8 & 37 & \\
\hline MRI enhancement & & & & 0.59 \\
\hline Homogeneous & 8 & 2 & 10 & \\
\hline Heterogeneous & 26 & 8 & 34 & \\
\hline \multicolumn{5}{|l|}{ MRI signal of tumor } \\
\hline TIWI & & & & 0.41 \\
\hline Isointense & 7 & 1 & 8 & \\
\hline Hypointense & 27 & 9 & 36 & \\
\hline T2WI & & & & 0.58 \\
\hline Isointense & 5 & 1 & 6 & \\
\hline Hyperintense & 29 & 9 & 38 & \\
\hline DWI & & & & 0.01 \\
\hline Moderate hyperintense & 24 & 2 & 26 & \\
\hline Marked hyperintense & 10 & 8 & 18 & \\
\hline P-duct dilation & 4 & 1 & 5 & 0.68 \\
\hline Lymph node or distal metastases & 0 & 2 & 2 & 0.05 \\
\hline Size & $2.2 \pm 1.6$ & $3.1 \pm 1.8$ & & 0.14 \\
\hline
\end{tabular}

MRI, magnetic resonance imaging; T1WI, T1-weighted image; PanNET, pancreatic neuroendocrine tumor; G1, grade 1.

duct. The ADC values were measured at least three times by each radiologist and the mean was calculated from the data obtained by the two radiologists.

Histological analysis. Pathological specimens were observed by light microscopy and immunohistochemical analysis. Briefly, the specimens were fixed in $4 \%$ paraformaldehyde at $4^{\circ} \mathrm{C}$ for $72 \mathrm{~h}$, dehydrated through a series of ascending ethanol solutions (40-100\%), embedded in paraffin and sliced at $5 \mu \mathrm{m}$ for hematoxylin and eosin staining. For immunohistochemical staining, the sections were incubated with 5\% BSA to block non-specific staining, and then incubated with an anti-Ki-67 monoclonal antibody (ab1667; dilution, 1:100; Abcam, Cambridge, MA, USA) overnight at $4^{\circ} \mathrm{C}$. The PanNETs were divided into PanNET G1 and PanNET G2, according to the updated WHO classification for neuroendocrine tumors, i.e., according to the mitotic incidence per 10 high-power fields (HPFs) or Ki-67 proliferation index (percentage of positive cells in areas of highest nuclear labeling). If the mitotic count was $<2 / 10 \mathrm{HPFs}$ and/or the Ki-67 index was $\leq 2$, the tumor was diagnosed as NET G1. If the mitotic count was 2-20/10 HPFs and/or the Ki-67 index was 3-20, the tumor was diagnosed as NET G2.

Statistical analysis. The data were analyzed using SPSS 16.0 (SPSS, Inc., Chicago, IL, USA) and Medcalc software (Ostend,
Belgium). Quantitative data were shown as the mean \pm standard deviation (SD) and were analyzed by an independent-sample t-test or one-way analysis of variance. Qualitative data were represented as percentages and were analyzed using the $\chi^{2}$ test or Fisher's exact test. Spearman's rank correlation analysis was adopted to assess the correlation between ADC values and histopathological variables. Receiver operating characteristic (ROC) curves were adopted to determine the cut-off values of ADC and its sensitivity, specificity and accuracy of prediction. $\mathrm{P}<0.05$ was considered to indicate a statistically significant difference.

\section{Results}

Patient characteristics. The characteristics of the PanNET patients are listed in Table I. Patient ages range from 30 to 76 years (mean \pm SD, 52.8 \pm 10.1 years). In total, $27(61.4 \%)$ patients were female and $17(38.6 \%)$ patients were male. Overall, 17 (38.6\%) patients were asymptomatic. Abdominal pain, confusion of consciousness, dizziness and hypodynamia was observed in 8, 5, 6 and 3 patients, respectively. According to the WHO classification, PanNET G1 tumors were observed in 34 patients $(77.3 \%)$ and PanNET G2 tumors were observed in 10 patients $(22.7 \%)$. A total of 23 lesions were observed in the pancreatic head and neck, and 21 lesions were observed in the body and tail. The size of the masses ranged from 0.8 to 

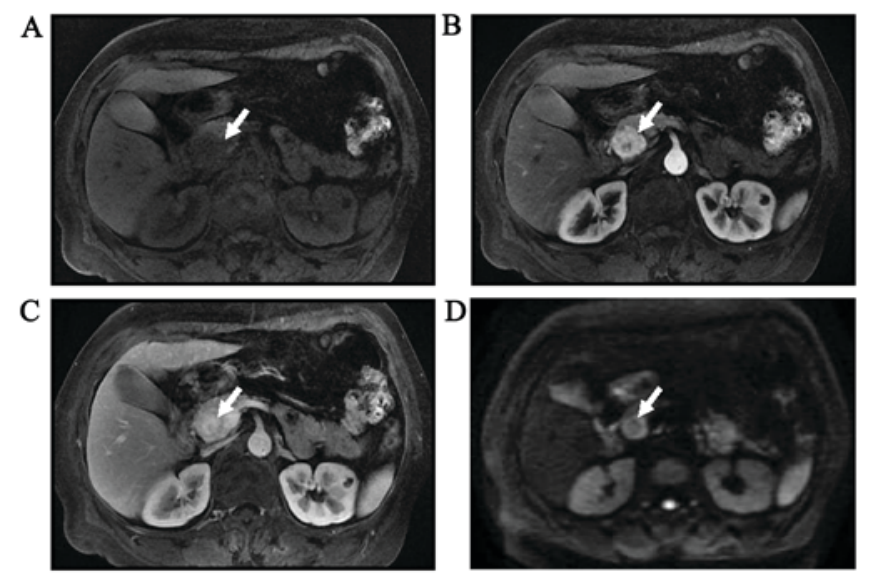

Figure 1. Representative case of a 65 -year-old female with a pathologically proven G1 neuroendocrine tumor in the pancreatic head. In (A) T1-weighted unenhanced and gadolinium-enhanced images in (B) the arterial phase and (C) the portal phase, the tumor (arrows) exhibits a hypointense signal with a well-defined border on the unenhanced image and appears as a higher enhancement compared with the normal pancreatic parenchyma in the arterial and portal phase. (D) In the DWI image $\left(b=1,000 \mathrm{sec} / \mathrm{mm}^{2}\right)$, the tumor appears as a hyperintense lesion compared with the normal pancreatic parenchyma.
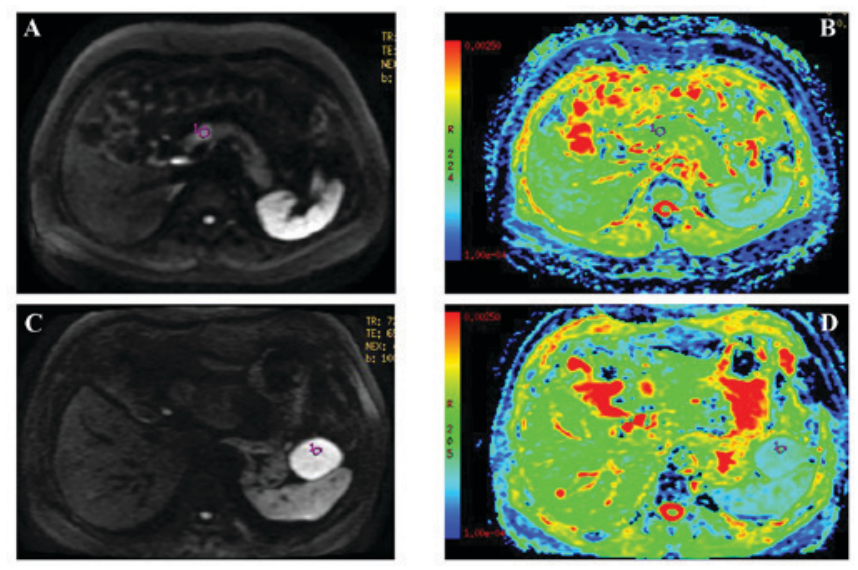

Figure 3. DWI and ADC map images of G1 and G2 tumors. (A) The DWI $\left(b=1,000 \mathrm{sec} / \mathrm{mm}^{2}\right)$ and (B) ADC map image of a 56-year-old female with a G1 neuroendocrine tumor. (C) The DWI $\left(1,000 \mathrm{sec} / \mathrm{mm}^{2}\right)$ and (D) ADC map image of a 50-year-old female with a G2 neuroendocrine tumor. Compared with the normal pancreatic parenchyma, the tumors appear as hyperintense lesions on DWI images. The ADC values are (B) $1.15 \times 10^{-3} \mathrm{~mm}^{2} / \mathrm{sec}$ and (D) $0.925 \times 10^{-3} \mathrm{~mm}^{2} / \mathrm{sec}$, respectively. DWI, diffusion weighted imaging; ADC, apparent diffusion coefficient.

$6.5 \mathrm{~cm}$, with a mean diameter of $2.4 \pm 1.6 \mathrm{~cm}$. The mean levels of carcinoembryonic antigen, cancer antigen 19-9 and blood glucose were $5.35 \mathrm{ng} / \mathrm{ml}$ (normal reference level, $<5 \mathrm{ng} / \mathrm{ml}$ ), $51.2 \mathrm{kU} / \mathrm{l}$ (normal reference level, <37 kU/l) and $4.9 \mathrm{mmol} / \mathrm{l}$ (normal reference level, $<6.1 \mathrm{mmol} / \mathrm{l}$ ), respectively.

MRI findings of PanNET G1 and PanNET G2. Table II summarizes the MRI findings of PanNET. A common MRI finding for PanNET G2 compared with PanNET G1 was ill-defined tumor borders $(\mathrm{P}=0.009)$. In total, 3 patients $(30.0 \%)$ with PanNET G2 exhibited tumors with ill-defined borders, whereas all PanNET G1 patients exhibited tumors
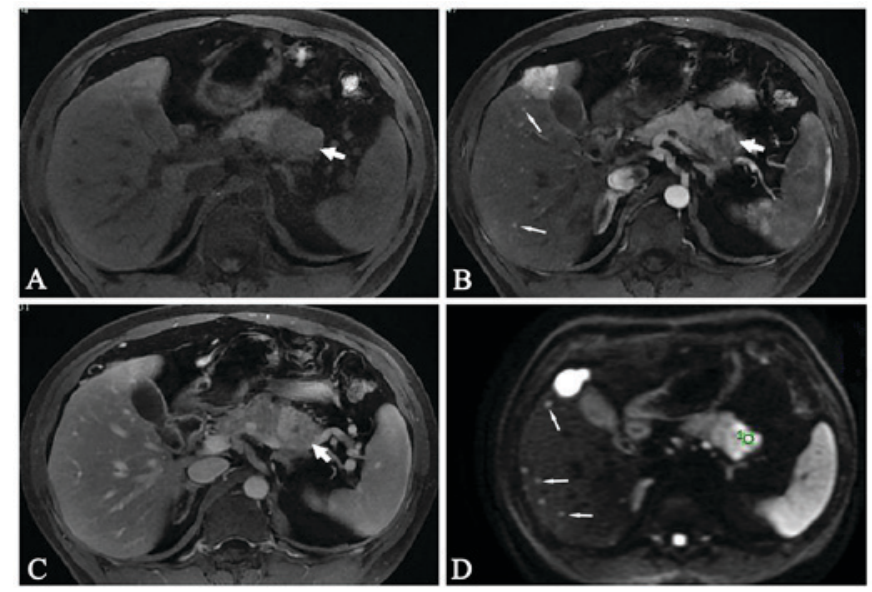

Figure 2. Representative case of a 54-year-old female with a pathologically proven G2 neuroendocrine tumor in the pancreatic body. In (A) T1-weighted unenhanced and gadolinium-enhanced images in (B) the arterial phase and (C) the portal phase, the tumor (arrows) exhibits a hypointense signal with a well-defined border on the unenhanced image and appears as a higher enhancement compared with the normal pancreatic parenchyma in the arterial and portal phase. (D) In the DWI image $\left(b=1,000 \mathrm{sec} / \mathrm{mm}^{2}\right)$, the tumor appears as a hyperintense lesion compared with the normal pancreatic parenchyma. Liver metastases can be observed on the arterial phase (B) image and DWI (D, long arrows).

with well-circumscribed borders. Lymph node metastases or distal metastases were more often observed in PanNET G2 compared with G1 tumors $(\mathrm{P}<0.05)$. No differences in solid and cystic pattern, enhancement, MRI signal of parenchyma, pancreatic duct dilation or size were observed between PanNET G1 and PanNET G2 tumors. A total of 10 patients with PanNET G1 and 8 patients with PanNET G2 showed marked hyperintensity in DWI images $(\mathrm{P}=0.008)$. The T1-weighted unenhanced and gadolinium-enhanced images of PanNET G1 and G2 are shown in Figs. 1 and 2.

The DWI and ADC maps of PanNET G1 and PanNET G2 are shown in Fig. 3. Fig. 4 shows mean ADC values, which are significantly different between the normal pancreas and PanNET G1 $(\mathrm{P}<0.01)$, between the normal pancreas and PanNET G2 $(\mathrm{P}<0.01)$, and between PanNET G1 and PanNET $\mathrm{G} 2(\mathrm{P}<0.05)$.

Correlation between ADC values and pathological parameters. Negative correlations were found between ADC values and the Ki-67 proliferation index $(\mathrm{r}=-0.132, \mathrm{P}=0.031)$, mitotic count $(\mathrm{r}=-0.124, \mathrm{P}=0.04)$ and $\mathrm{PanNET}$ grade $(\mathrm{r}=-0.159$, $\mathrm{P}=0.006)$.

ROC analysis. ROC curves were used to determine the cut-off values that differentiate PanNET G1 from PanNET G2 (Fig. 5). The area under the curve (AUC) is 0.81 . The cut-off value is $0.930 \times 10^{-3} \mathrm{~mm}^{2} / \mathrm{sec}$, with $82.4 \%$ sensitivity and $79.5 \%$ specificity for predicating PanNET G2 tumors.

\section{Discussion}

Determination of the lesion grade prior to surgical resection would allow for the appropriate treatment to be administered. Several imaging approaches have been used to discriminate between presumably benign and malignant PanNET. However, 


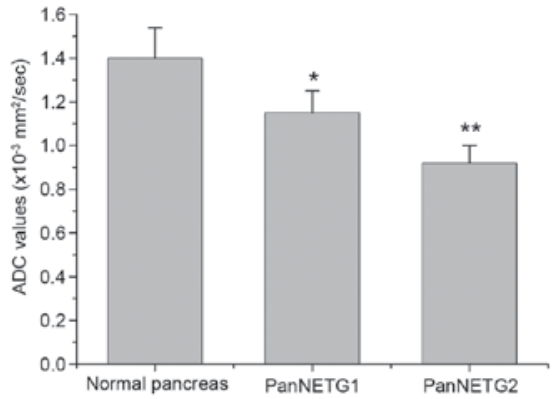

Figure 4. ADC values of normal pancreatic parenchyma, PanNET G1 and PanNET G2. Significant differences in mean ADC values were observed between normal pancreatic parenchyma and G1 and G2 tumors. ${ }^{*} \mathrm{P}<0.01$ by independent-sample t-test, compared with normal pancreatic parenchyma; ${ }^{* *} \mathrm{P}<0.05$ by one-way analysis of variance, compared with $\mathrm{G} 1$ tumor and normal pancreatic parenchyma. ADC, apparent diffusion coefficient; PanNET, pancreatic neuroendocrine tumor; G1, grade 1.

few studies have used imaging methods to differentiate between PanNET G1 and PanNET G2 (2). In the present study, the data showed that ADC values correlate with the Ki-67 proliferation index, mitotic count and PanNET grade. The findings in the study further demonstrated that DWI can be used to discriminate between PanNET G1 and PanNET G2, with acceptable sensitivity and specificity.

PanNETs are considered to be hypervascular tumors. Therefore, contrast-enhanced CT and MRI have been used to discriminate between presumably benign and malignant PanNET $(5,11,12)$. Cappelli et al (11) demonstrated that the CT contrast enhancement pattern may preoperatively associate with PanNET behavior and WHO grade. Kim et al (12) showed that NEC could be differentiated from G1/G2 by using enhanced CT. Gadoxetic-acid-enhanced MRI also revealed differences in enhancement pattern in the arterial phase between benign and non-benign PanNETs (5). Wang et al (1) and Jang et al (5) previously used DWI to assess differences in ADC values between PanNET subtypes and observed a significant difference in mean ADC between well-differentiated PanNET and NEC. PET/CT can also be used for the preoperative differential diagnosis of PanNETs of varying grades (13). The maximum standardized uptake value (SUVmax) of NEC tumors is significantly higher than that of G1/G2 tumors, which suggests that FDG-PET is useful for differentiating NEC PanNETs from G1/G2 PanNETs (13). However, these studies mainly showed the differential diagnosis of benign and non-benign PanNETs.

Certain G2 tumors also have metastatic features (13). Therefore, the differentiation between G1 and G2 tumors should also be made prior to surgical resection. Several studies have been conducted to discriminate between G1 and G2 tumors using imaging methods $(1,2,9)$. Takumi et al (9) showed that enhancements in contrast in the pancreatic parenchymal and portal venous phase differ significantly between G1 and G2 tumors. Kim et al (2) reported that ADC values differed significantly between PanNET G1 and G2, and that the ADC value was useful for differentiating PanNET G1 from G2. Wang et al (1) showed that the ADC value of G2 tumors was lower than that of G1 tumors. The results of the present study are consistent with Kim et al (2) and Wang et al (1). A significant difference was observed between ADC values

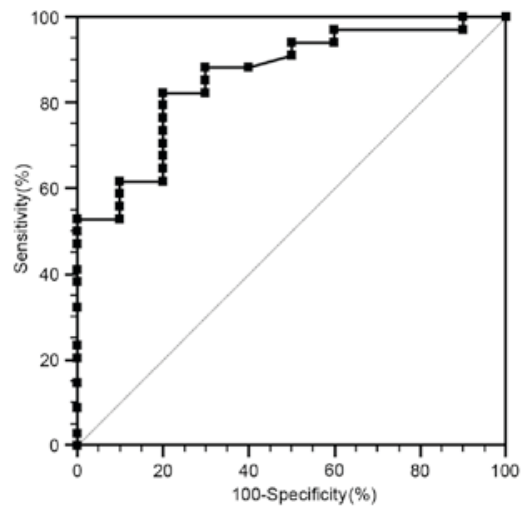

Figure 5. Receiver operating characteristic curve of the mean apparent diffusion coefficient value used to differentiate pancreatic neuroendocrine tumor G1 and G2 tumors. The area under the curve is 0.81 .

for PanNET G1 and G2. Using ADC values to distinguish G1 from G2 exhibited a sensitivity of $82.4 \%$ and specificity of $79.5 \%$, with an AUC of 0.81 . The data further confirmed that the ADC value is also associated with the grade of the well-differentiated PanNET.

The ADC value could reflect the degree of water motion (1). The diffusion of water, dense cellularity and extracellular space tortuosity are recognized as the reasons for the decrease in ADC value in malignant tumors. Previous data have shown that the cell density of G2 tumors is higher than that of G1 tumors, which may result in a decrease in the volume of extracellular and intracellular spaces (1). Consequently, the free motion of water molecules is restricted, resulting in a decrease in ADC values (18). In addition, the increased tumor cellularity could also cause the decrease in ADC value (16). Wang et al (1) speculated that marked fibrosis may also account for the low ADC values of certain well-differentiated PanNETs.

On the basis of the WHO PanNET grading classification, the mitotic count and Ki-67 proliferation index have been recognized as critical pathological parameters $(7,8)$. PanNETs are classified as G1 if they have a mitotic count $<2$ per $10 \mathrm{HPFs}$ and/or a Ki-67 index $<2 \%$, as G2 if they have a mitotic count of 2-20 per $10 \mathrm{HPFs}$ and/or a Ki-67 index of 3-20\%, and as NEC if they have a mitotic count $>20$ per $10 \mathrm{HPFs}$ and/or a Ki-67 index $>20 \%$. Higher Ki-67 labeling index values are associated with high-grade malignancy. The present study additionally showed that ADC value is also associated with histopathological factors, including mitotic count and Ki-67 proliferation index.

This study had several potential limitations. First, as PanNETs are rare, the number of patients was relatively small, particularly for the PanNET G2 patients, which may limit the statistical power. As such, further studies with large sample sizes will be required. Second, patients with NEC are not included in this study, as such tumors were considered unresectable (19). Third, as the results of ROC analysis are associated with patient population, the data may be different to other studies and therefore requires validation by future studies. Finally, as this is a retrospective study, selection bias may exist.

In conclusion, the present data demonstrated that well-differentiated PanNET with different WHO classification grades have varying ADC values. ADC value also correlates 
with the pathological parameters of PanNET (the mitotic count and $\mathrm{Ki}-67$ index). ADC value is therefore a valuable imaging parameter in predicting WHO grade in well-differentiated PanNET.

\section{References}

1. Wang Y, Miller FH, Chen ZE, Merrick L, Mortele KJ, Hoff FL, Hammond NA, Yaghmai V and Nikolaidis P: Diffusion-weighted MR imaging of solid and cystic lesions of the pancreas. Radiographics 31: E47-E64, 2011.

2. Kim JH, Eun HW, Kim YJ, Han JK and Choi BI: Staging accuracy of MR for pancreatic neuroendocrine tumor and imaging findings according to the tumor grade. Abdom Imaging 38: 1106-1114, 2013.

3. Ehehalt F, Saeger HD, Schmidt CM and Grützmann R: Neuroendocrine tumors of the pancreas. Oncologist 14: 456-467, 2009.

4. Ito T, Sasano H, Tanaka M, Osamura RY, Sasaki I, Kimura W, Takano K, Obara T, Ishibashi M, Nakao K, et al: Epidemiological study of gastroenteropancreatic neuroendocrine tumors in Japan. J Gastroenterol 45: 234-243, 2010

5. Jang KM, Kim SH, Lee SJ and Choi D: The value of gadoxetic acid-enhanced and diffusion-weighted MRI for prediction of grading of pancreatic neuroendocrine tumors. Acta Radiol 55: 140-148, 2014.

6. DeLellis RA, Lloyd RV and Heitz P, Eng C (eds.): Pathology and genetics of tumors of endocrine organs. International Agency for Research on Cancer (IARC), Lyon, pp177-182, 2004.

7. Klimstra DS, Arnold R and Capella C: Neuroendocrine neoplasms of the pancreas. In: Bosman FT, Carneiro F, Hruban RH, Theise ND (eds). WHO Classification of Tumours of the Digestive System. Vol 3. 4th edition. International Agency for Research on Cancer (IARC), Lyon, pp322-326, 2010.

8. Bosman FT, Carneiro F, Hruban RH and Theise N: WHO classification of tumours of the digestive system. Lyon: IARC Press, 2010.

9. Takumi K, Fukukura Y, Higashi M, Ideue J, Umanodan T, Hakamada H, Kanetsuki I and Yoshiura T: Pancreatic neuroendocrine tumors: Correlation between the contrast-enhanced computed tomography features and the pathological tumor grade. Eur J Radiol 84: 1436-1443, 2015.
10. Rindi G and Wiedenmann B: Neuroendocrine neoplasms of the gut and pancreas: New insights. Nat Rev Endocrinol 8: 54-64, 2012.

11. Cappelli C, Boggi U, Mazzeo S, Cervelli R, Campani D, Funel N, Contillo BP and Bartolozzi C: Contrast enhancement pattern on multidetector CT predicts malignancy in pancreatic endocrine tumours. Eur Radiol 25: 751-759, 2015.

12. Kim DW, Kim HJ, Kim KW, Byun JH, Song KB, Kim JH and Hong SM: Neuroendocrine neoplasms of the pancreas at dynamic enhanced CT: Comparison between grade 3 neuroendocrine carcinoma and grade 1/2 neuroendocrine tumour. Eur Radiol 25: 1375-1383, 2015.

13. Tomimaru Y, Eguchi H, Tatsumi M, Kim T, Hama N, Wada H, Kawamoto K, Kobayashi S, Morii E, Mori M, et al: Clinical utility of 2-[(18)F] fluoro-2-deoxy-Dglucose positron emission tomography in predicting World Health Organization grade in pancreatic neuroendocrine tumors. Surgery 157: 269-276, 2015.

14. Balci NC, Perman WH, Saglam S, Akisik F, Fattahi R and Bilgin M: Diffusion-weighted magnetic resonance imaging of the pancreas. Top Magn Reson Imaging 20: 43-47, 2009.

15. Wang Y, Chen ZE, Yaghmai V, Nikolaidis P, McCarthy RJ, Merrick L and Miller FH: Diffusion-weighted $\mathrm{mr}$ imaging in pancreatic endocrine tumors correlated with histopathologic characteristics. J Magn Reson Imaging 33: 1071-1079, 2011.

16. Higano S, Yun X, Kumabe T, Watanabe M, Mugikura S, Umetsu A, Sato A, Yamada T and Takahashi S: Malignant astrocytic tumors: Clinical importance of apparent diffusion coefficient in prediction of grade and prognosis. Radiology 241: 839-846, 2006

17. Humphries PD, Sebire NJ, Siegel MJ and Olsen ØE: Tumors in pediatric patients at diffusion-weighted MR imaging: Apparent diffusion coefficient and tumor cellularity. Radiology 245: 848-854, 2007

18. Padhani AR, Liu G, Koh DM, Chenevert TL, Thoeny HC, Takahara T, Dzik-Jurasz A, Ross BD, Van Cauteren M, Collins D, et al: Diffusion-weighted magnetic resonance imaging as a cancer biomarker: Consensus and recommendations. Neoplasia 11: 102-125, 2009.

19. Tatsumoto S, Kodama Y,Sakurai Y, Shinohara T, Katanuma A and Maguchi H: Pancreatic neuroendocrine neoplasm: Correlation between computed tomography enhancement patterns and prognostic factors of surgical and endoscopic ultrasound-guided fine-needle aspiration biopsy specimens. Abdom Imaging 38: 358-366, 2013. 